\title{
Sélection de variétés de blé dur (Triticum durum Desf.) et de blé tendre (Triticum aestivum L.) adaptées à la sécheresse par la mesure de l'extinction de la fluorescence de la chlorophylle
} in vivo

\author{
Michel HAVAUX, Mohamed ERNEZ \& Robert LANNOYE \\ Laboratoire de Physiologie végétale, Université Libre de Bruxelles, 28, avenue Paul Heger, 1050 Bruxelles, \\ Belgique
}

RÉSUMÉ

\begin{abstract}
La fluorescence de la chlorophylle a été mesurée dans les feuilles de 25 variétés et de 8 nouveaux génotypes de blé dur (Triticum durum Desf.) ainsi que dans 3 variétés de blé tendre (Triticum aestivum L.) so'.mises à une déshydratation rapide de $4 \mathrm{~h}$ à l'air libre. La déshydratation foliaire a modifié l'aspect des courbes d'induction de la fluorescence chlorophyllienne. En particulier, la décroissance de la fluorescence du niveau maximum $\mathbf{P}$ à l'état pseudo-stationnaire S (mesurée par le rapport (P-S)/S) a été inhibée. Cette inhibition a toutefois été fortement dépendante du degré de tolérance à la sécheresse des différentes variétés de blé : dans les variétés sensibles, le facteur (P-S)/S a été spectaculairement réduit alors que dans les variétés très tolérantes, il a été peu modifié. De même, la composante photochimique de l'extinction de la fluorescence chlorophyllienne $\left(\mathrm{q}_{\mathrm{Q}}\right)$, déterminée à l'état stationnaire au moyen d'une technique de fluorescence modulée, a été plus ou moins affectée suivant le degré de résistance des variétés de blé. Les résultats présentés dans cet article ont montré que des paramètres de fluorescence simples à mesurer comme le rapport (P-S)/S ou $q_{0}$ peuvent être utilisés en pratique pour diagnostiquer rapidement la résistance à la sécheresse des génotypes de blé dur ou de blé tendre.
\end{abstract}

Mots clés additionnels : Induction de la fluorescence chlorophyllienne, photosynthèse, potentiel hydrique.

Screening of varieties of durum wheat (Triticum durum Desf.) and bread wheat (Triticum aestivum L.) for drought adaptation by measuring in vivo chlorophyll fluorescence quenching.

Chlorophyll fluorescence was measured in leaves of 25 varieties and 8 new genotypes of durum wheat (Triticum durum Desf.) and 3 varieties of bread wheat (Triticum aestivum L.), submitted to rapid desiccation in air for $4 \mathrm{~h}$. Leaf dehydration modified the shape of the chlorophyll fluorescence induction curves. In particular, fluorescence quenching from the maximum level $\mathrm{P}$ to the pseudo-steady state $\mathrm{S}$ (estimated by the (P-S)/S ratio) was inhibited. However, this inhibition was shown to be strongly dependent on the drought tolerance of the wheat varieties : in sensitive varieties, the (P-S)/S ratio was spectacularly reduced whereas it remained almost unchanged in very tolerant varieties. Similarly, the photochemical component of the chlorophyll fluorescence quenching $\left(\mathrm{q}_{\mathrm{Q}}\right)$, determined at the steady state using a modulated fluorescence technique, was more or less affected, depending on the drought tolerance level of the wheat varieties. The results presented in this paper indicated that easily measurable fluorescence parameters, such as $(\mathrm{P}-\mathrm{S}) / \mathrm{S}$ or $\mathrm{q}_{\mathrm{Q}}$, could be used in practice to estimate rapidly the resistance of wheat genotypes to drought conditions.

Additional key words : Chlorophyll fluorescence induction, photosynthesis, water potential.

\section{INTRODUCTION}

Les pigments des feuilles végétales (chlorophylles, caroténoïdes) absorbent la lumière dont l'énergie est utilisée dans les réactions photochimiques primaires de la photosynthèse. Une partie de l'énergie lumineuse absorbée est toutefois dissipée sous forme d'émission de fluorescence et de chaleur. La mesure de la fluores- 
cence de la chlorophylle qui, aux températures physiologiques, provient essentiellement du photosystème II (PS II), fournit de précieux renseignements sur le fonctionnement de l'appareil photochimique des chloroplastes (SCHREIBER, 1983 ; KRAUSE \& WEIS, 1984 ; BRIANTAIS et al., 1986). En effet, lorsqu'une feuille est adaptée à l'obscurité et ensuite ramenée à la lumière, le rendement de la fluorescence chlorophyllienne varie suivant une cinétique relativement complexe (augmentation rapide de l'intensité de la fluorescence suivie d'une diminution lente vers un état stationnaire) due à l'activation progressive des mécanismes photosynthétiques (ce phénomène d'induction de la fluorescence est appelé " effet Kautsky », voir KAUTSKY et al., 1960). Cette fluorescence variable, observable uniquement dans les tissus photosynthétiquement actifs, dépend de divers processus photochimiques tels que l'état redox des accepteurs primaires d'électrons du PS II, l'établissement du gradient de $\mathrm{pH}$ photoinduit dans les chloroplastes, etc..., qui peuvent ainsi être étudiés indirectement.

Lorsque l'état fonctionnel des membranes photosynthétiques se dégrade sous l'effet de certaines contraintes défavorables de l'environnement comme la chaleur ou le froid par exemple, l'altération des processus photosynthétiques se reflète dans les courbes d'induction de la fluorescence de la chlorophylle (SMILliE \& GibBONS, 1981 ; HeTHERINGTHON et al., 1983 ; HAVAUX \& LANNOYE, 1984 et $1985 c$; RENGER \& SCHREIBER, 1986). Il a été suggéré que certains paramètres, calculés à partir des courbes de fluorescence, pourraient être utilisés en pratique afin de détecter facilement des conditions de stress dans les plantes et de mesurer rapidement leur degré de résistance aux contraintes de l'environnement (SMILLIE \& HETHERINGTON, 1983 ; HETHERINGTHON et al., 1983 ; HAVAUX \& LANNOYE, 1985a).

La sécheresse est un facteur écologique qui perturbe également le mécanisme photosynthétique de transfert d'électrons (BOYER \& BOWEN, 1970 ; HAVAUX et al., $1986 b$; GENTY et al., 1987). Les effets d'un arrêt de l'irrigation de diverses espèces végétales ont pu être suivis in vivo, notamment par la mesure de l'amplitude de la fluorescence variable (GOVINDJEE et al., 1981 ; HavauX \& LANNOYE, 1983 ; Havaux et al., 1987). Dans une expérience préliminaire utilisant un nombre limité de variétés de blé dur soumises à une déshydratation foliaire rapide, nous avons observé que la vitesse de décroissance de la fluorescence chlorophyllienne vers l'état stationnaire (mesurée par la pente maximum de la décroissance) pourrait éventuellement être utilisée pour établir un test de sélection rapide de variétés de blé dur résistantes à la sécheresse (HAVAuX \& LANNOYE, 1985b). L'étude présentée dans cet article a pour but d'examiner en détail cette hypothèse séduisante : nous avons soumis un grand nombre de variétés et de nouveaux génotypes de blé dur, ainsi que quelques variétés de blé tendre, à une dessiccation rapide et nous avons ensuite mesuré la fluorescence émise par ces feuilles. Les résultats indiquent que l'extinction de la fluorescence chlorophyllienne in vivo est un bon indicateur de la tolérance à la sécheresse chez le blé et que ce paramètre pourrait servir dans les programmes de sélection de variétés de blé adaptées aux conditions de sécheresse.

\section{MATÉRIEL ET MÉTHODES}

\section{A. Matériel végétal}

Un grand nombre de variétés commerciales de blé dur (Triticum durum Desf.) ont été examinées. La liste de ces variétés est donnée dans les tableaux 1 et 2 .

\section{TABLEAU 1}

Effet de la déshydratation pendant $4 \mathrm{~h}$ d'échantillons de feuilles de $2 I$ variétés de blé dur et de 3 variétés de blé tendre (Arz, Dougga $B$. et FlorencexAurore) sur l'extinction de la fluorescence de la chlorophylle (de $P$ à $S)$, mesurée par le rapport $(P-S) / S$. Le potentiel hydrique des feuilles traitées a été de $-17 \pm 2$ bars pour toutes les variétés à l'exception de FlorencexAurore, Poinville et Tomclair dont le potentiel hydrique a été de -27 bars. Potentiel hydrique des témoins $=-4 \pm 1$ bars.

Effect of $4 \mathrm{~h}$ dehydration of leaf samples of 21 durum wheat varieties and 3 bread wheat varieties (Arz, Dougga B. and FlorencexAurore) on chlorophyll fluorescence quenching (from $P$ to S), measured by the $(P-S) / S$ ratio. The water potential of the stressed leaves was $-17 \pm 2$ bar in all the varieties except FlorencexAurore, Poinville and Tomclair in which the leaf water potential was -27 bar. Water potential of the controls $=-4 \pm l \mathrm{bar}$.

\begin{tabular}{|c|c|c|c|}
\hline \multirow{2}{*}{ Variétés } & \multicolumn{3}{|c|}{$(\mathrm{P}-\mathrm{S}) / \mathrm{S}$} \\
\hline & Témoins & & Traités \\
\hline \multicolumn{4}{|c|}{ Très tolérantes à la sécheresse } \\
\hline Oued Zenati & $0,39 \pm 0,07^{\mathrm{a}}$ & 0,38 & $\pm 0,08(100 \%)$ \\
\hline Polonicum & $0,69 \pm 0,05$ & 0,59 & $\pm 0,03(85 \%)$ \\
\hline Hedba 3 & $0,54 \pm 0,12$ & 0,45 & $\pm 0,05(83 \%)$ \\
\hline Arz & $0,58 \pm 0,04$ & 0,46 & $\pm 0,05(79 \%)$ \\
\hline Dougga $\mathrm{B}$ & $0,58 \pm 0,07$ & 0,44 & $\pm 0,12(76 \%)$ \\
\hline Aziza & $0,51 \pm 0,07$ & 0,36 & $\pm 0,05(70 \%)$ \\
\hline Gloire de Mongolfier & $0,78 \pm 0,06$ & 0,54 & $\pm 0,07(69 \%)$ \\
\hline Tassili & $0,58 \pm 0,05$ & 0,37 & $\pm 0,06(64 \%)$ \\
\hline \multicolumn{4}{|l|}{ Moyennement tolérantes } \\
\hline Khroub & $0,72 \pm 0,06$ & 0,41 & $\pm 0,10(57 \%)$ \\
\hline Mahon Demias & $0,74 \pm 0,11$ & 0,42 & $\pm 0,22(57 \%)$ \\
\hline Rekhem & $0,64 \pm 0,05$ & 0,34 & $\pm 0,22(53 \%)$ \\
\hline Waha & $0,70 \pm 0,05$ & 0,36 & $\pm 0,09(51 \%)$ \\
\hline Maghrebi & $0,69 \pm 0,05$ & 0,35 & $\pm 0,06(51 \%)$ \\
\hline Inia 66 & $0,53 \pm 0,06$ & 0,27 & $\pm 0,10(51 \%)$ \\
\hline Sahel & $0,61 \pm 0,04$ & 0,29 & $\pm 0,08(47 \%)$ \\
\hline Mondur & $0,64 \pm 0,05$ & 0,25 & $\pm 0,06(39 \%)$ \\
\hline KKB & $0,82 \pm 0,13$ & 0,30 & $\pm 0,06(37 \%)$ \\
\hline Tell & $0,62 \pm 0,03$ & 0,23 & $\pm 0,07(37 \%)$ \\
\hline \multicolumn{4}{|l|}{ Sensibles } \\
\hline Timgad & $0,74 \pm 0,06$ & 0,21 & $\pm 0,05(28 \%)$ \\
\hline Hd $1220 \mathrm{Kalb}$ & $0,70 \pm 0,07$ & 0,13 & $\pm 0,12(18 \%)$ \\
\hline Poinville & $0,69 \pm 0,06$ & 0,10 & $\pm 0,04(14 \%)$ \\
\hline Tomclair & $0,72 \pm 0,05$ & 0,09 & $\pm 0,03(12 \%)$ \\
\hline Cando & $0,64 \pm 0,04$ & 0,07 & $\pm 0,05(11 \%)$ \\
\hline FlorencexAurore & $0,66 \pm 0,07$ & 0,07 & $\pm 0,07(11 \%)$ \\
\hline
\end{tabular}

a Les valeurs données dans le tableau sont les moyennes de 5 mesures \pm déviation standard. Entre parenthèses : valeurs des traités en $\%$ des témoins.

Trois variétés de blé tendre (Triticum aestivum L.), Arz, Dougga B. et FlorencexAurore, ont également été utilisées à des fins de comparaison. De par leur origine (française, nord-africaine, mexicaine), ces cultivars possèdent différents degrés de tolérance à la sécheresse. Des études physiologiques (portant notamment sur l'effet de conditions de sécheresse sur la concentration foliaire en proline libre, la résistance foliaire à la transpiration, la turgescence cellulaire et le rendement) menées à la Station d'Amélioration des Plantes de l'Ecole Nationale Supérieure Agronomique 
TABLEAU 2

Effet de la déshydratation pendant $4 \mathrm{~h}$ de feuilles de cultivars de blé sur la composante photochimique $q_{Q}$ de l'extinction de la fluorescence chlorophyllienne.

Effect of 4-h dehydration of leaf samples of wheat cultivars on the photochemical component $q_{Q}$ of chlorophyll fluorescence quenching.

\begin{tabular}{|c|c|c|c|}
\hline \multirow{2}{*}{$\begin{array}{l}\text { Noms des variétés } \\
\text { et des génotypes }\end{array}$} & \multicolumn{3}{|c|}{$\mathrm{q}_{\mathrm{Q}}$} \\
\hline & Témoins & & Traités \\
\hline \multicolumn{4}{|l|}{ Tolérants à la sécheresse } \\
\hline Waha & $0,90^{\mathrm{a}}$ & 0,86 & $(96 \%)$ \\
\hline Bidi 17 & 0,91 & 0,872 & $(96 \%)$ \\
\hline Arz & 0,89 & 0,84 & $(94 \%)$ \\
\hline Hedba 3 & $0,70 \pm 0,01$ & $0,65 \pm$ & $=0,15(93 \%)$ \\
\hline Mohamed Ben Bachir & 0,90 & 0,83 & $(92 \%)$ \\
\hline Tassili & 0,90 & 0,81 & $(90 \%)$ \\
\hline Khroub & 0,91 & 0,81 & $(89 \%)$ \\
\hline $28-4-2$ & $0,66 \pm 0,01$ & $0,52 \pm$ & $=0,06(79 \%)$ \\
\hline \multicolumn{4}{|l|}{ Sensibles } \\
\hline 408-7 & $0,69 \pm 0,01$ & $0,52 \pm$ & $=0,08(75 \%)$ \\
\hline 36 & $0,64 \pm 0,03$ & $0,47 \pm$ & $=0,04(73 \%)$ \\
\hline $522-2-7$ & $0,76 \pm 0,01$ & $0,53 \pm$ & $=0,02(70 \%)$ \\
\hline 1710 & $0,75 \pm 0,01$ & $0,48 \pm$ & $=0,04(64 \%)$ \\
\hline Clairdoc & $0,79 \pm 0,03$ & $0,49 \pm$ & $=0,10(62 \%)$ \\
\hline Tomclair & $0,89 \pm 0$ & $0,52 \pm$ & $=0,01(58 \%)$ \\
\hline 37 & $0,83 \pm 0,01$ & $0,45 \pm$ & $=0,04(54 \%)$ \\
\hline Poinville & 0,89 & 0,46 & $(52 \%)$ \\
\hline KKB & 0,88 & 0,44 & $(50 \%)$ \\
\hline Durelle & 0,89 & 0,39 & $(44 \%)$ \\
\hline $37-7-7$ & $0,77 \pm 0,01$ & $0,29 \pm$ & $0,01(37 \%)$ \\
\hline $30-1-2$ & $0,69 \pm 0,10$ & $0,19 \pm$ & $=0,07(27 \%)$ \\
\hline
\end{tabular}

a Les valeurs présentées dans ce tableau sont soit les moyennes de trois expériences \pm déviation standard, soit les résultats d'une expérience. Entre parenthèses : valeurs des feuilles traitées exprimées en \% des témoins.

de Montpellier ont permis de caractériser la plupart de ces variétés du point de vue de leur adaptation aux conditions de déficit hydrique (MONNEVEUX \& NEMMAR, 1986 ; P. GRIGNAC, comm. pers., 1987) : le degré d'adaptation à la sécheresse des variétés utilisées s'est échelonné de la très forte tolérance (par exemple, la variété Hedba 3 ou Oued Zenati) à la très grande sensibilité (par exemple, Tomclair ou Durelle). Nous avons également utilisé divers génotypes $(36,37,28-4$ $2,408-7,522-2-7,1710,30-1-2$ et 37-7-7), sélectionnés récemment à la Station d'Amélioration des Plantes de Montpellier, dont les caractéristiques de tolérance à la sécheresse sont encore inconnues.

Les plantules ont été cultivées en serre dans des vases de culture cylindriques (diamètre, $30 \mathrm{~cm}$ ) remplis de terreau et arrosés journellement. Les mesures de fluorescence ont été réalisées sur des échantillons de feuilles pris sur des plantules ayant atteint le stade « 4 feuilles » après 3-4 semaines de croissance.

\section{B. Traitement des échantillons de feuille}

Des segments rectangulaires (de $2 \mathrm{~cm}$ de long) ont été prélevés sur la troisième feuille bien développée. Ces échantillons ont ensuite été soumis, avant les mesures de fluorescence, à un traitement identique à celui utilisé par HAVAUX \& LANNOYE (1985b) et HAVAUX et al. (1986a) : les échantillons ont été déshydratés rapidement en les plaçant à l'obscurité sur du papier filtre (Whatman $\mathrm{n}^{\circ} 1$ ) pendant plusieurs heures (en général $4 \mathrm{~h}$ ), les témoins étant conservés sur du papier filtre imbibé d'eau distillée. La température et l'humidité relative de l'air pendant le traitement ont été respectivement de $26^{\circ} \mathrm{C}$ et de $55 \%$. Après ce traitement, le potentiel hydrique $\psi$ des feuilles déshydratées et des témoins a été mesuré en utilisant une presse à membrane (RENARD \& NDAYISHIMIE, 1982). Ces mesures de potentiel hydrique foliaire ont montré que les cinétiques de déshydratation ont été, à peu de choses près, identiques dans toutes les variétés de blé utilisées, à l'exception toutefois de trois variétés particulièrement sensibles à la sécheresse (FlorencexAurore, Poinville et Tomclair) qui ont atteint des potentiels hydriques notablement plus négatifs pour un même temps de déshydratation ( -27 bars au lieu de - 17 bars après $4 \mathrm{~h}$ de déshydratation).

\section{Fluorescence de la chlorophylle}

Les courbes d'induction de la fluorescence de la chlorophylle ont été mesurées au moyen d'un fluorimètre portable décrit par SCHREIBER et al. (1975) et commercialisé par RICHARD BRANCKER RESEARCH (Ottawa, Canada; Plant Productivity Fluorometer, Model SF-10). La fluorescence chlorophyllienne émise par un échantillon de feuille, préalablement adapté à l'obscurité pendant environ $30 \mathrm{~min}$, a été induite par une lumière rouge $\left(670 \mathrm{~nm}, 15 \mu \mathrm{E} \mathrm{m}^{-2} \mathrm{~s}^{-1}\right)$ produite par une petite diode émettrice. La fluorescence a été détectée aux longueurs d'onde supérieures à $710 \mathrm{~nm}$ par une photodiode réceptrice protégée par un filtre passe-haut. Le signal de fluorescence a été digitalisé et analysé par un ordinateur COMMODORE 64 connecté à un convertisseur $\mathrm{A} / \mathrm{D}$ construit au laboratoire et décrit en détail par PANNEELS et al. (1987).

La composante photochimique de l'extinction de la fluorescence de la chlorophylle a été déterminée au moyen d'un nouveau type de fluorimètre imaginé par SCHREIBER et al. (1986) et construit par la firme WALZ (Effeltrich, RFA ; modèle PAM 101-102-103). La fluorescence (modulée) a été induite par de courtes impulsions de lumière rouge $(1 \mu \mathrm{s})$ appliquées à une fréquence de $1,6 \mathrm{kHz}$. Cette lumière d'excitation, produite par une diode émettrice de lumière $(650 \mathrm{~nm})$, a eu une très faible intensité (valeur intégrée $=0,5 \mathrm{nE}$ $\mathrm{m}^{-2} \mathrm{~s}^{-1}$ ) de telle sorte qu'aucun phénomène d'induction n'a pu être induit. Elle a donc permis de mesurer le niveau initial de la fluorescence $F_{0}$ (un exemple de courbe de fluorescence mesurée au moyen de ce fluorimètre est présenté à la fig. 4). L'induction de la fluorescence chlorophyllienne a été induite par une seconde lumière, blanche, non modulée et de plus forte intensité $\left(1000 \mu \mathrm{E} \mathrm{m}^{-2} \mathrm{~s}^{-1}\right)$ produite par une lampe halogène de $150 \mathrm{~W}$ (Osram Xenoplot HLX). L'induction a été mesurée avec une fréquence de modulation de $110 \mathrm{kHz}$ de manière à réduire fortement le bruit de fond parasite et à augmenter sensiblement le temps de réponse de l'appareil (SCHREIBER et al., 1986). Le niveau maximum de la fluorescence de la chlorophylle a été déterminé par l'adjonction d'un éclair $(1 \mathrm{~s})$ de lumière blanche de très forte intensité, saturante pour le transfert d'électrons $(8000 \mu \mathrm{E}$ $\mathrm{m}^{-2} \mathrm{~s}^{-1}$ ). Dans cette étude, les mesures de fluorescence ont été réalisées sur des échantillons de feuilles exposés à de l'air contenant $0,03 \%$ de $\mathrm{CO}_{2}$. La méthode de calcul utilisée pour estimer la composante 
photochimique de l'extinction de fluorescence, à partir du niveau initial, stationnaire et maximum de la fluorescence, est expliquée dans la partie Résultats et Discussion.

Toutes les densités de flux de photons ont été mesurées à l'aide d'un radiomètre $\mathrm{Li}$-Cor $188 \mathrm{~B}$.

\section{RÉSULTATS ET DISCUSSION}

La figure 1 (première courbe, en haut) montre un exemple typique de courbe d'induction de la fluorescence de la chlorophylle émise par une feuille de blé dur (var. Tell) non stressée, préalablement adaptée à l'obscurité. Au moment de l'éclairement, l'intensité de la fluorescence augmente quasi instantanément jusqu'à un niveau initial (appelé $\mathrm{O}$ ), correspondant à un état où tous les accepteurs primaires d'électrons $\left(Q_{A}\right)$ du photosystème II (PS II) sont oxydés. Ensuite, la fluorescence augmente rapidement (dans l'intervalle d'une seconde environ) du niveau $O$ jusqu'à un niveau maximum P. Cette augmentation rapide de la fluorescence reflète la réduction de $\mathrm{Q}_{\mathrm{A}}$ (KRAUSE \& WEIS, 1984 ; BRIANTAIS et al., 1986). La fluorescence chlorophyllienne ne reste cependant pas bloquée à ce niveau $\mathrm{P}$ mais décroît lentement vers un état stationnaire plus bas $(\mathrm{T})$ en passant généralement par un second maximum intermédiaire $M$. Cette lente décroissance (3-4 min) de la fluorescence est un phénomène complexe attribué à divers facteurs dont la réoxydation des $\mathrm{Q}_{\bar{A}}^{-}$réduits (composante photochimique de l'extinction de la fluorescence), l'établissement du gra-

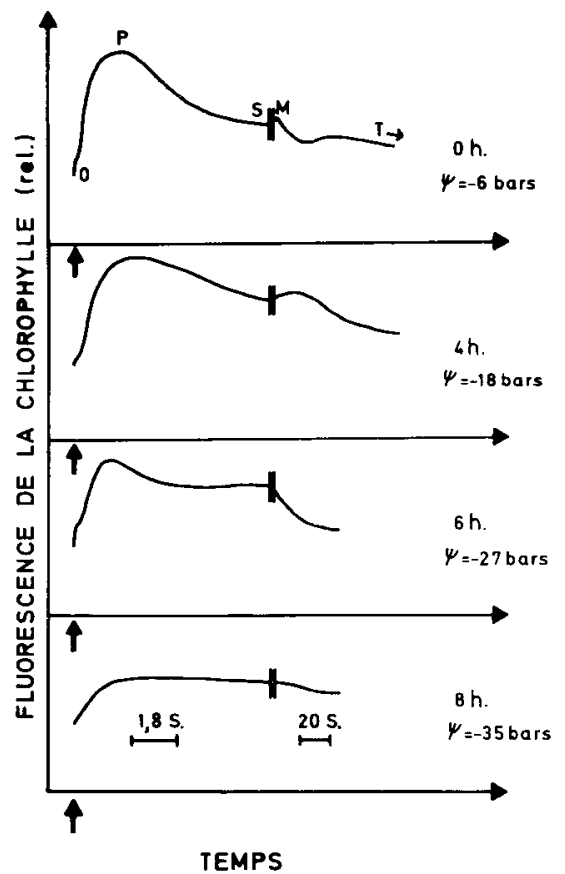

Figure 1

Courbes d'induction de la fluorescence de la chlorophylle émise par des morceaux de feuilles de blé dur (var. Tell) soumis à une déshydratation rapide à l'air pendant 0, 4, 6 et $8 \mathrm{~h}$. Les échantillons de feuille, préalablement adaptés à l'obscurité pendant environ $30 \mathrm{~min}$, ont été brusquement illuminés au moment indiqué par la flèche (temps 0 de l'induction).

Chlorophyll fluorescence induction curves of durum wheat leaf samples (var. Tell) submitted to rapid dehydration in air for 0, 4, 6 and $8 \mathrm{~h}$. The leaf samples, previously dark-adapted for around $30 \mathrm{~min}$, were suddenly illuminated at the time indicated by the arrow. dient de pH transthylacoïdien (composante énergétique) et le phénomène de transition état $1 /$ état 2 au cours duquel l'énergie d'excitation est redistribuée entre les deux photosystèmes au profit du PS I (moins fluorescent que le PS II) (KRAUSE \& WEIS, 1984 ; BRIANTAIS et al., 1986 ; SCHREIBER et al., 1986).

Lorsque la feuille se déshydrate (fig. 1), l'aspect de la courbe d'induction se modifie sensiblement. L'inhibition de la décroissance de la fluorescence (de $\mathrm{P}$ à $\mathrm{S}$, $S$ étant le niveau pseudo-stationnaire avec le second pic $\mathrm{M}$ ) apparaît comme le changement le plus marqué. Ce phénomène peut être quantifié en calculant le rapport (P-S)/S. La figure 2 montre l'évolution de la valeur de ce rapport au cours de la déshydratation de feuilles de la variété Tell : on peut observer une considérable et rapide diminution de $(\mathrm{P}-\mathrm{S}) / \mathrm{S}$ en réponse à la contrainte hydrique. L'amplitude de la fluorescence variable $((\mathrm{P}-\mathrm{O}) / \mathrm{O})$, qui est un indicateur de l'activité de transfert d'électrons du PS II, a également diminué mais uniquement pour les fortes dessiccations foliaires (durée du traitement $>4 \mathrm{~h}$ ). Ce paramètre de fluorescence, qui s'est révélé être très sensible à la plupart des contraintes de l'environnement, tels la chaleur (SMillie \& GibBons, 1981), le froid (HETHERINGTHON et al., 1983 ; HAVAUX \& LANNOYE, 1984) ou les radiations ultra-violettes (SMILLIE, 1982), semble donc être nettement moins sensible au déficit hydrique que la phase lente (P-S-M-T) de décroissance de la fluorescence.

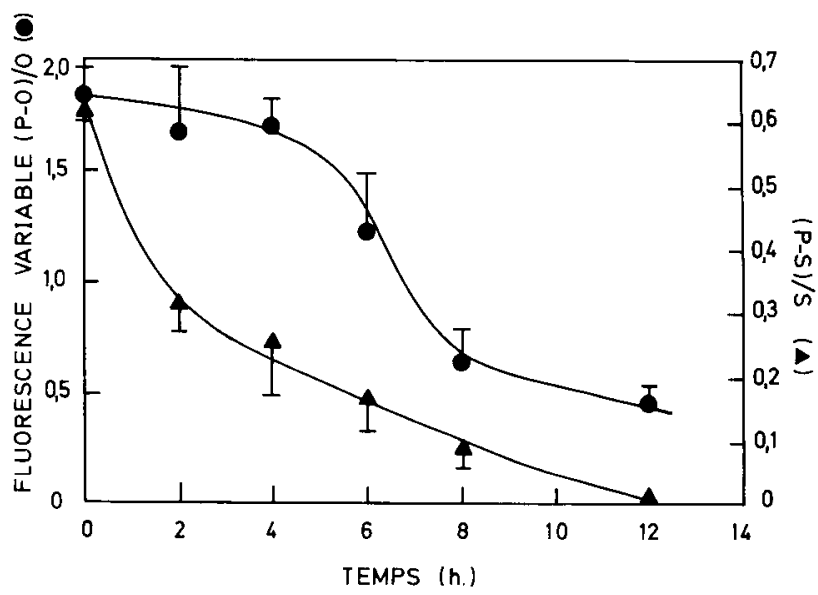

Figure 2

Amplitude de la fluorescence variable $(P-O) / O$ et rapport $(P-S) / S$ déterminés à partir des courbes d'induction de la fluorescence de la chlorophylle de feuilles de blé dur (var. Tell) exposées à la déshydratation. Les points $O, P$ et $S$ sont définis à la figure 1 . Les déviations standards (écarts-types) des moyennes $(n=5)$ sont représentées par les barres verticales.

Amplitude of the variable fluorescence $(P-O) / O$ and $(P-S) / S$ ratio value determined from the chlorophyll fluorescence induction curves of durum wheat leaves (var. Tell) exposed to dehydration in air. The $O, P$ and $S$ levels are defined in figure 1. The standard deviation of the means $(n=5)$ are shown by the vertical lines.

La figure 3 montre que le rapport (P-S)/S est linéairement relié au potentiel hydrique foliaire $\psi$ (tout au moins pour les potentiels hydriques supérieurs à - 30 bars). Il est intéressant de remarquer également que la pente de la droite de la variété Tell (moyennement tolérante à la sécheresse) diffère de celle de la variété Dougga B. (très tolérante à la sécheresse). 


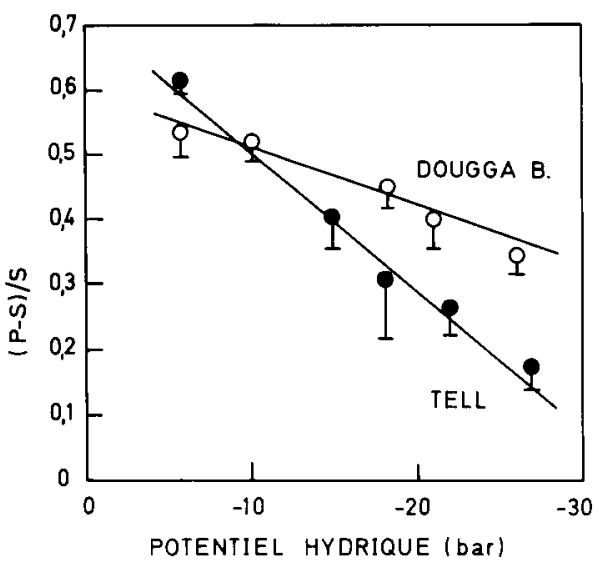

Figure 3

Corrélations entre la décroissance de la fluorescence de la chlorophylle de $P$ à $S$ (quantifiée par le rapport $(P-S) / S$ ) et le potentiel hydrique des feuilles de deux variétés de blé, Tell et Dougga B. Barres verticales $=$ déviations standards (écart-type).

Correlation between the chlorophyll fluorescence quenching from $P$ to $S$ (measured by $(P-S) / S$ ratio) and the leaf water potential in two wheat varieties. Tell and Dougga B. Vertical lines = standard deviations.

Pour une même valeur du potentiel hydrique foliaire, l'inhibition de la phase P-S de l'induction de la fluorescence chlorophyllienne a été beaucoup moins forte pour la variété très tolérante que pour la variété moyennement tolérante. Le rapport (P-S)/S apparaît donc comme un indicateur intéressant (et facile à mesurer) du stress hydrique dans les feuilles de blé ainsi que du degré de tolérance à la sécheresse de la variété considérée.

Afin de tester cette idée, les feuilles de 21 variétés de blé dur et de 3 variétés de blé tendre, possédant différents degrés connus de tolérance à la sécheresse, ont été soumises à une déshydratation rapide pendant $4 \mathrm{~h}$ et le rapport (P-S)/S a été mesuré avant et après ce traitement (tabl. 1). Les résultats ont indiqué une considérable diversité chez le blé en ce qui concerne l'inhibition de la phase lente P-S de l'induction de la fluorescence. Dans les feuilles des variétés connues pour être très tolérantes à la sécheresse, le rapport $(\mathrm{P}-\mathrm{S}) / \mathrm{S}$ n'a été que faiblement réduit après le traitement (en moyenne, $25 \%$ de diminution). Par contre, les variétés sensibles au stress hydrique se sont caractérisées par une inhibition spectaculaire de l'extinction de la fluorescence (environ $85 \%$ d'inhibition). Entre ces deux classes de plantes, un troisième groupe intermédiaire, correspondant aux variétés faiblement résistantes à la sécheresse, a pu être distingué. Ces résultats indiquent qu'un paramètre photosynthétique simple à mesurer comme le rapport (P-S)/S peut servir de base pour établir un test rapide de sélection de variétés de blé résistantes à la sécheresse. L'appareillage nécessaire est relativement simple et même portable (voir, SCHREIBER et al., 1975; MELCAREK et al., 1977). La mesure est rapide puisque l'extinction de la fluorescence de $\mathrm{P}$ à $\mathrm{S}$ dure seulement quelques secondes (voir fig. 1). Cependant, le principal inconvénient de cette technique est la nécessité d'adapter les feuilles à l'obscurité pendant un laps de temps relativement long de manière à oxyder tous les $\mathrm{Q}_{\mathrm{A}}$ avant l'éclairement de la feuille. Cette complication réduit évidemment la possibilité de cribler un très grand nombre d'échantillons et empêche les mesures en plein champ.

Récemment, SCHREIBER et al. (1986) ont développé une nouvelle technique de fluorescence modulée permettant de séparer et d'estimer les différentes composantes (composante photochimique et composante non photochimique) de l'extinction de la fluorescence chlorophyllienne; des variantes de cette méthode ont également été proposées par ÖGREN \& BAKER (1985) et QUiCK \& HORTON (1984). La figure 4 résume le principe de la mesure de l'extinction photochimique de la fluorescence (appelée $\mathrm{q}_{\mathrm{Q}}$ ) au moyen de cette technique. La fluorescence modulée (niveau $\mathrm{F}_{0}$ correspondant à $100 \%$ de $Q_{A}$ oxydés) est excitée par une lumière hachée ayant une très faible intensité de manière à ne pas induire l'effet Kautsky. L'induction de la fluorescence (niveau $F_{v}$ ) est créé par l'addition d'une seconde lumière plus forte non modulée. Le niveau maximum de fluorescence $\left(F_{0}+F_{v}+\Delta F\right)$, correspondant à $100 \%$ de $\mathrm{Q}_{\mathrm{A}}^{-}$réduits, est obtenu par l'addition d'une lumière continue saturante. La décroissance de la fluorescence chlorophyllienne due à la réoxydation de $\mathrm{Q}_{\mathrm{A}}^{-}\left(\mathrm{q}_{\mathrm{Q}}\right)$ peut alors être calculée par le rapport suivant : $\Delta \mathrm{F} /\left(\mathrm{F}_{\mathrm{v}}+\Delta \mathrm{F}\right)$ (SCHREIBER et al., 1986). Cette méthode permet donc de mesurer $\mathrm{q}_{\mathrm{Q}}$ en n'importe quel point de la courbe d'induction et notamment à l'état stationnaire, c'est-à-dire dans une feuille adaptée (et photosynthétisant) à une certaine intensité lumineuse. Il n'est donc plus nécessaire

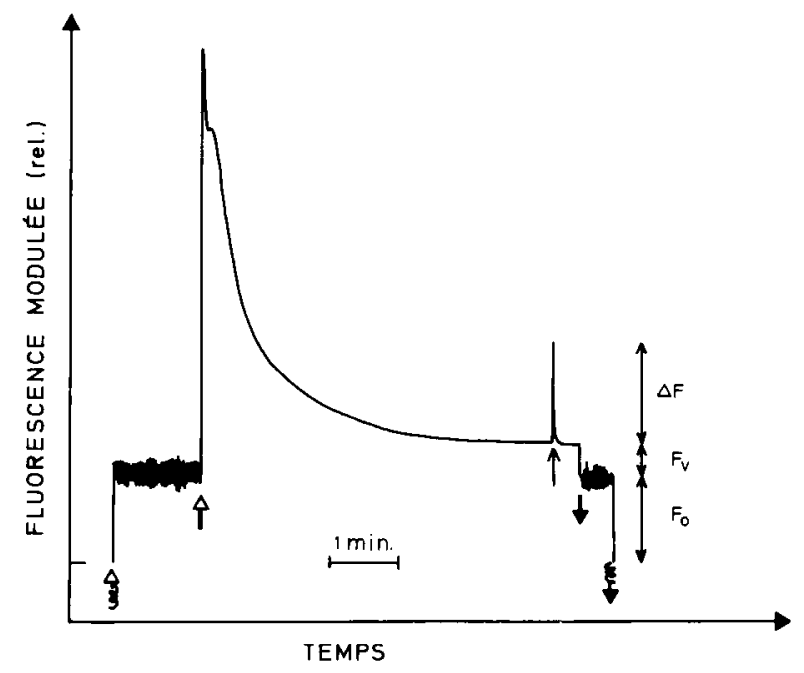

Figure 4

Mesure de la composante photochimique $\left(q_{Q}\right)$ de la décroissance de la fluorescence de la chlorophylle dans une feuille (non stressée) de blé dur (var. Clairdoc). La feuille (dans ce cas-ci, adaptée à l'obscurité) a d'abord été illuminée avec une très faible lumière modulée de manière à déterminer le niveau 0 de la fluorescence $\left(F_{o}\right)$ (s), lumière modulée allumée; éteinte). La fluorescence variable $\left(F_{\nu}\right)$ a été induite au moyen d'une seconde lumière (non modulée et de plus forte intensité ; $\uparrow$, allumée ; $\downarrow$, éteinte). L'adjonction d'un éclair de lumière saturante (1) a induit une augmentation de la fluorescence $(\Delta F)$ jusqu'au niveau maximum. A partir des valeurs de $F_{o}$, $F_{v}$ et $\Delta F, q_{O}$ peut être calculé en utilisant la formule suivante $q_{Q}=\Delta F /\left(\Delta F+F_{v}\right)$.

Photochemical quenching ( $q_{\alpha}$ ) of chlorophyll fluorescence in nonstressed durum wheat leaf (var. Clairdoc). The leaf (in this case. dark-adapted) was first illuminated with a dim modulated light in order to measure the 0 level of fluorescence $\left(F_{\partial}\right)$ (sodulated light on; off). The variable fluorescence $(F)$ was induced by a second light (non-modulated and having a higher intensity; $\uparrow$, on; $\downarrow$ off). Addition of a pulse of saturating light (1) resulted in a fluorescence rise $(\Delta F) . q_{Q}$ can be calculated from the $F_{\omega}, F_{v}$ and $\Delta F$ levels using the following formula $q_{Q}=\Delta F /\left(\Delta F+F_{\nu}\right)$. 
d'adapter à l'obscurité les feuilles avant la mesure. D'autre part, du fait qu'il s'agit d'une technique modulée, les lumières non modulées ne créent pas par elles-mêmes de signal de fluorescence modulée (en réalité, le système de détection synchrone n'enregistre pas les signaux non modulés) et les mesures peuvent donc se faire en champ en présence de la lumière solaire.

Le tableau 2 montre les résultats d'une expérience au cours de laquelle $\mathrm{q}_{\mathrm{Q}}$ a été mesuré (à l'état stationnaire) dans plusieurs variétés connues de blé (utilisées comme références) ainsi que dans un certain nombre de génotypes inconnus. On peut clairement distinguer les variétés tolérantes à la sécheresse de celles qui sont sensibles : alors que, chez les variétés résistantes, $\mathrm{q}_{\mathrm{Q}} \mathrm{a}$ été très peu affecté par la déshydratation foliaire, une notable inhibition de l'extinction photochimique a été observée chez les variétés sensibles. Une distinction claire a également pu être faite parmi les huit génotypes examinés : un nouveau génotype adapté aux conditions de sécheresse (28-4-2) a en effet été détecté et séparé des génotypes apparemment très sensibles (377-7 et 30-1-2) au moyen des mesures de fluorescence. Il faut toutefois remarquer que l'inhibition de $q_{Q}$ induite par la contrainte hydrique a été nettement moins forte que celle de la décroissance totale de la fluorescence (mesurée entre $P$ et $S$ par le rapport $(\mathrm{P}-\mathrm{S}) / \mathrm{S})$ : par exemple, $(\mathrm{P}-\mathrm{S}) / \mathrm{S}$ et $\mathrm{q}_{\mathrm{Q}}$ ont été réduits respectivement à 12 et $58 \%$ des témoins chez le cultivar Tomclair déshydraté. Les différences entre variétés sont donc moins marquées en utilisant la diminution de $\mathrm{q}_{\mathrm{Q}}$ comme indicateur de résistance. Cela s'explique vraisemblablement par le fait que les deux phénomènes sont différents. Dans un cas (phase P-S), bien qu'il ait été suggéré que la réoxydation de $Q_{A}^{-}$ soit le processus essentiel déterminant la décroissance du rendement de la fluorescence au cours de cette phase (BRADBURY \& BAKER, 1981 ; BRIANTAIS et al., 1986), le rapport (P-S)/S est une mesure globale (et finalement peu rigoureuse) de l'extinction de la fluorescence. Dans l'autre cas (état stationnaire), de nombreux processus participent à l'extinction de la fluorescence. La méthode utilisée (SCHREIBER et al., 1986) a permis une mesure plus correcte de l'extinction de la

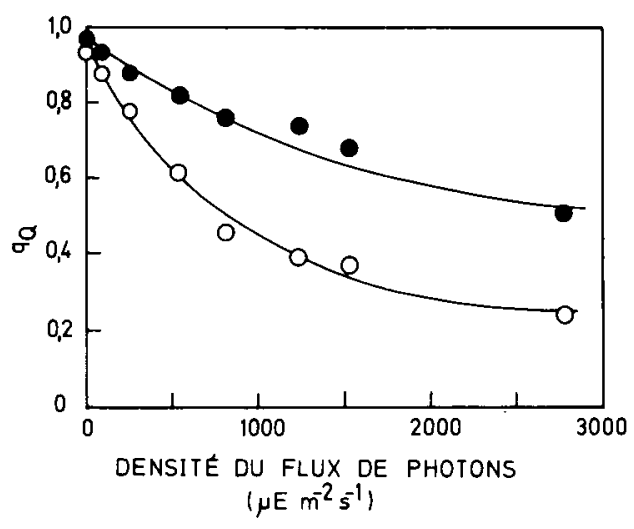

Figure 5

Composante photochimique $q_{O}$ de l'extinction de la fluorescence de la chlorophylle dans des feuiltes de blé dur (génotype 1710) déshydratées à l'air pendant $0(\bullet, \psi=-5$ bars $)$ et $4 \mathrm{~h}(\mathrm{o}$, $\psi=-19$ bars).

Photochemical component $q_{g}$ of chlorophyll fluorescence in durum wheat leaves (genotype 1710$)$ dehydrated in air for $01 \mathbf{0}$, $\psi=-5$ bar $)$ and $4 h(o, \psi=-19$ bar $)$. fluorescence en séparant et quantifiant une des composantes $\left(\mathrm{q}_{\mathrm{Q}}\right)$ du phénomène. D'autre part, il convient de signaler que les mesures de (P-S)/S et de $\mathrm{q}_{0}$ ont été faites dans des conditions expérimentales différentes. En effet, la phase P-S a été analysée sur des courbes d'induction obtenues au moyen d'une lumière rouge dont l'intensité a été relativement faible $\left(15 \mu \mathrm{E} \mathrm{m} \mathrm{m}^{-2}\right.$ $\mathrm{s}^{-1}$, intensité maximum de la photodiode) alors que l'extinction photochimique de la fluorescence $q_{r}$ a été mesurée avec une lumière blanche de plus forte intensité $\left(1000 \mu \mathrm{E} \mathrm{m}^{-2} \mathrm{~s}^{-1}\right)$. Cette intensité élevée a été utilisée pour amplifier l'effet de la déshydratation foliaire sur la valeur de $\mathrm{q}_{\mathrm{Q}}$. En effet, comme le montre la figure 5 , la différence entre feuilles déshydratées et témoins a été beaucoup plus limitée aux faibles densités de flux photonique (inférieurs à $200 \mu \mathrm{E} \mathrm{m}^{-2} \mathrm{~s}^{-1}$ par exemple) qu'aux densités de flux supérieures à environ $1000 \mu \mathrm{E} \mathrm{m} \mathrm{m}^{-2} \mathrm{~s}^{-1}$.

\section{CONCLUSIONS}

Les résultats présentés dans cet article confirment l'intérêt des mesures de la fluorescence de la chlorophylle in vivo pour l'étude de l'adaptation des plantes cultivées aux contraintes du milieu. Dans le cas particulier des contraintes hydriques, nous avons montré que la conservation de l'activité d'extinction de la fluorescence (ou tout du moins de la composante photochimique de cette extinction) dans les feuilles rapidement déshydratées est caractéristique de plantes tolérantes à la sécheresse et peut ainsi servir d'indicateur simple et rapide de la tolérance relative à la sécheresse chez le blé et probablement chez les céréales en général. Bien que les mesures faites dans la présente étude concernent la tolérance à la déshydratation foliaire et ne tiennent donc pas compte d'éventuels mécanismes d'adaptation (ajustement osmotique, par exemple), le classement obtenu correspond parfaitement à celui fourni par des mesures agronomiques en plein champ. Les mesures de fluorescence chlorophyllienne in vivo peuvent être partiellement automatisées en utilisant un ordinateur connecté au fluorimètre par l'intermédiaire d'un digitaliseur, tel que, par exemple, celui décrit par NoRRISH et al. (1983) ou PANNEELS et al. (1987), permettant de stocker et de traiter un très grand nombre de données. De cette manière, la méthode du $\mathrm{q}_{\mathrm{Q}}$ présentée dans cet article pourrait être un outil efficace pour les sélectionneurs intéressés dans le criblage de plantes cultivées résistantes à la sécheresse.

Reçu le 5 octobre 1987 Accepté le 27 décembre 1987.

\section{REMERCIEMENTS}

Nous remercions le Professeur P. Grignac pour nous avoir offert les semences des différentes variétés et génotypes de blé utilisés dans cette étude. M. Havaux remercie le Fonds National de la Recherche Scientifique (Belgique) pour l'attribution d'un mandat de Chargé de Recherches. Ce travail de recherche a été financé par un contrat CEE (AGRIMED) $n^{\circ}$ VI/4741/84-F(VIPF4/501). 


\section{RÉFÉRENCES BIBLIOGRAPHIQUES}

Boyer J. S., Bowen B. L., 1970. Inhibition of oxygen evolution in chloroplasts isolated from leaves with low water potentials. Plant Physiol., 45, 612-615.

Bradbury M., Baker N. R., 1981. Analysis of the slow phases of the in vivo chlorophyll fluorescence induction curve. Changes in the redox state of photosystem II electron acceptor and fluorescence emission from photosystems I and II. Biochim. Biophys. Acta, 635, 542-551.

Briantais J.-M., Vernotte C., Krause G. H., Weis E., 1986. Chlorophyll $a$ fluorescence of higher plants : chloroplasts and leaves, p. 539-584. In Govindjee, J. Amesz \& D. C. Fork. Light emission by plants and bacteria. Academic Press, N.Y.

Genty B., Briantais J.-M., Vieira da Silva J. B., 1987. Ëfects of drought on primary photosynthetic processes of cotton leaves. Plant Physiol., 83, 360-364

Govindjee, Downton W. J. S., Fork D. C., Armond P. A., 1981. Chlorophyll $a$ fluorescence transient as an indicator of water potential of leaves. Plant Sci. Lett., 20, 191-194.

Havaux M., Lannoye R., 1983. Chlorophyll fluorescence induction : a sensitive indicator of water stress in maize plants. Irrig. Sci., 4, 147-151.

Havaux M., Lannoye R., 1984. Effects of chilling temperatures on prompt and delayed chlorophyll fluorescence in maize and barley leaves. Photosynthetica, 18, 117-127.

Havaux M., Lannoye R., 1985a. In vivo chlorophyll fluorescence and delayed light emission as rapid screening techniques for stress tolerance in crop plants. Z. Pflanzenzüchtg., 95, 1-13.

Havaux M., Lannoye R., 1985b. Drought resistance of hard wheat cultivars measured by a rapid chlorophyll fluorescence test. $J$. Agric. Sci., Camb., 104, 501-504.

Havaux M., Lannoye R., 1985c. Effets des basses températures positives sur les réactions photochimiques primaires de la photosynthèse du maïs (Zea mays L., cv. « LG 9 »). Agronomie, 5, 331-337.

Havaux M.. Canaani O., Malkin S.. 1986a. Photosynthetic responses of leaves to water stress, expressed by photoacoustics and related methods. I. Probing the photoacoustic method as an indicator for water stress in vivo. Plant Physiol., 82, 827-833.

Havaux M., Canaani O., Malkin S., 1986b. Photosynthetic responses of leaves to water stress, expressed by photoacoustics and related methods. II. The effect of rapid drought on the electron transport and the relative activities of the two photosystems. Plant Physiol., 82, 834-839.

Havaux M., Canaani O., Malkin S., 1987. Inhibition of photosynthetic activities under slow water stress measured in vivo by the photoacoustic method. Physiol. Plant., 70, 503-510.

Hetheringthon S. E., Smillie R. M., Hardacre A. K., Eagles H. A., 1983. Using chlorophyll fluorescence in vivo to measure the chilling tolerance of different populations of maize. Aust. J. Plant. Physiol., 10, 247-256.

Kautsky H., Appel W., Aman H., 1960. Chlorophyll-Fluorescenz und Kohlensäureassimilation : die Fluorescenzkurve und die Photochemie der Pflanze. Biochem. Z., 322, 277-292.
Krause G. H., Weis E., 1984. Chlorophyll fluorescence as a tool in plant physiology. Il. Interpretation of fluorescence signals. Photosynthesis Res., 5, 139-157.

Melcarek P. K., Cernohlavek L. G., Brown G. N., 1977. A solidstate device for the simultaneous measurement of prompt and delayed chlorophyll fluorescence induction transients in leaves. Anal. Biochem., 82, 473-484.

Monneveux P., Nemmar M., 1986. Contribution à l'étude de la résistance à la sécheresse chez le blé tendre (Triticum aestivum L.) et chez le blé dur (Triticum durum Desf.) : étude de l'accumulation de la proline au cours du cycle de développement. Agronomie, 6, 583-590.

Norrish R., Kriedemann P. E., Wiskich J. T., 1983. Chlorophyll $a$ fluorescence transients : a fast data acquisition system to facilitate in vivo measurements. Photosynthesis Res., 4, 213-227.

Ögren E., Baker N. R., 1985. Evaluation of a technique for the measurement of chlorophyll fluorescence from leaves exposed to continuous white light. Plant Cell Environ., 8, 539-547.

Panneels P., Van Moer A., Reimer P., Salis P., Chouhiat A., Lannoye R., Figeys H., 1987. Fluorescence investigation of DCMU and S-triazine herbicide activity in crop and weed species, p. 827-830. In J. Biggins. Progress in photosynthesis research, vol. 3. Martinus Nijhoff Publishers, Dordrecht

Quick W. P., Horton P., 1984. Studies on the induction of chlorophyll fluorescence in barley protoplasts. II. Resolution of fluorescence quenching by redox state and the transthylakoid $\mathrm{pH}$ gradient. Proc. R. Soc. Lond. B, 220, 371-382.

Renard C., Ndayishimie V., 1982. Etude des relations hydriques chez Coffea arabica L. I. Comparaison de la presse à membrane et de la chambre à pression pour la mesure du potentiel hydrique foliaire ( $\psi)$. Café Cacao Thé, 26, 27-30.

Renger G., Schreiber U., 1986. Practical applications of fluorometric methods to algae and higher plant research, p. 587-619. In Govindjee, J. Amesz \& D. C. Fork. Light emission by plants and bacteria. Academic press, N. Y.

Schreiber U., 1983. Chlorophyll fluorescence as a tool in plant fluorometer for measurement of chlorophyll fluorescence induction in plants. Rev. Sci. Instrum., 46, 538-542.

Schreiber U., 1983. Chlorophyll fluorescence as a tool in plant physiology. I. The measuring system. Photosynthesis Res., 4, 361373.

Schreiber U., Schliwa U., Bilger W., 1986. Continuous recording of photochemical and non-photochemical fluorescence quenching with a new type of modulation fluorometer. Photosynthesis Res., 10, 5162.

Smillie R. M., 1982. Chlorophyll fluorescence in vivo as a probe for rapid measurement of tolerance to ultraviolet radiation. Plant Sci. lett., 28, 283-289.

Smillie R. M., Gibbons G. C., 1981. Heat tolerance and heat hardening in crop plants measured by chlorophyll fluorescence. Carlsberg Res. Commun., 46, 395-403.

Smillie R. M., Hetheringthon S. E., 1983. Stress tolerance and stress-induced injury in crop plants measured by chlorophyll fluorescence in vivo. Plant Physiol., 72, 1043-1050. 